\title{
Lipofilling after breast conserving surgery: a comprehensive literature review investigating its oncologic safety
}

\author{
Stephanie Cohen ${ }^{1}$, Yurie Sekigami ${ }^{1}$, Theresa Schwartz ${ }^{2}$, Albert Losken ${ }^{3}$, Julie Margenthaler ${ }^{4}$, \\ Abhishek Chatterjee ${ }^{1}$
}

${ }^{1}$ Division of Plastic Surgery, Division of Surgical Oncology, Department of Surgery, Tufts Medical Center, Boston, MA, USA; ${ }^{2}$ Division of Surgical Oncology, Department of Surgery, St. Louis University Hospital, St. Louis, MO, USA; ${ }^{3}$ Division of Plastic Surgery, Department of Surgery, Emory University Hospital, Atlanta, GA, USA; ${ }^{4}$ Division of Endocrine and Oncologic Surgery, Department of Surgery, Barnes-Jewish Hospital, St. Louis, MO, USA

Contributions: (I) Conception and design: A Chatterjee, S Cohen; (II) Administrative support: A Chatterjee; (III) Provision of study materials or patients: None; (IV) Collection and assembly of data: S Cohen, Y Sekigami; (V) Data analysis and interpretation: All Authors; (VI) Manuscript writing: All authors; (VII) Final approval of manuscript: All authors.

Correspondence to: Abhishek Chatterjee, MD, MBA. Division of Plastic Surgery, Division of Surgical Oncology, Department of Surgery, Tufts Medical Center, South Building, 4th Floor, 800 Washington St., Box 1043, Boston, MA 02111, USA. Email: AChatterjee1@TuftsMedicalCenter.org.

\begin{abstract}
Lipofilling has regenerative properties used to improve deformities after breast conserving surgery. Our hypothesis is that there is inadequate data to ensure that lipofilling does not increase locoregional cancer recurrence after breast conserving surgery. A PRISMA comprehensive literature review was conducted of articles published prior to October 2019 investigating recurrence in patients who underwent lipofilling after breast conserving surgery. All forms of breast conserving surgery, fat grafting, and injection intervals were included. Patients undergoing mastectomy were excluded. Requirements to define lipofilling as "safe" included (I) a defined interval between resection and lipofilling; (II) a minimum followup period of 6 years from tumor resection; (III) a minimum follow-up period of 3 years from lipofilling; (IV) presence of a control group; (V) controls matched for ER/PR/Her-2; (VI) a sub-group analysis focusing on ER/PR/Her-2; (VII) adequate powering. Nineteen studies met inclusion criteria. The range in time from breast conserving surgery to fat injection was $0-76$ months. The average time to follow-up after lipofilling was 23 days-60 months. Two studies had a sufficient follow-up time from both primary resection and from lipofilling. Seventeen of the nineteen studies specified the interval between resection and lipofilling, but there is currently no consensus regarding how soon lipofilling can be performed following BCS. Eight studies performed a subgroup analysis in cases of recurrence and found recurrence after lipofilling was associated with number of positive axillary nodes, intraepithelial neoplasia, high grade histology, Luminal A subtype, age $<50$, Ki-67 expression, and lipofilling within 3 months of primary resection. Of the eleven studies that included a comparison group, one matched patient for Her-2 and there was a statistically significant difference in Her-2 positive cancers in the study arms of two articles. Several studies deemed lipofilling "safe," two showed association of lipofilling and local recurrence, and most studies concluded that further research was needed. Insufficient and contradictory data exists to demonstrate the safety of lipofilling after breast conserving surgery. A multicentered, well designed study is needed to verify the safety of this practice.
\end{abstract}

Keywords: Breast conserving surgery; lipofilling; recurrence

Submitted Jul 05, 2019. Accepted for publication Sep 27, 2019.

doi: 10.21037 /gs.2019.09.09

View this article at: http://dx.doi.org/10.21037/gs.2019.09.09 


\section{Introduction}

Breast conservation surgery (BCS) continues to be the most popular form of breast cancer surgery (1). As the indications for BCS continue to expand, there has been increased interest in personalized techniques of breast reconstruction (2). First introduced by Bircoll et al. in 1987, lipofilling, or autologous fat grafting, is a technique in which a patient's fat is harvested and centrifuged to obtain a concentrate rich in stem cells which is then transferred to the breast (3). With its advantages that include improved aesthetics in the area of the partial mastectomy, this technique is gaining increased use in BCS without clear consensus in this technique's oncologic safety. Multiple in vitro studies have conjectured that adipocytes, preadipocytes, and their products may play a role in tumor recurrence as they are involved in the tumor cell cycle through autocrine, paracrine, and exocrine/endocrine secretions (4,5). Moreover, a review article published by Lohsiriwat et al. proposed that a "tumor-stroma interaction" can potentially induce cancer reappearance by "fueling" dormant breast cancer cells in the tumor bed (6). This would be particularly concerning if it were to occur at a positive tumor margin after BCS.

In 2009, the American Society of Plastic Surgeons (ASPS) established a task force to assess the indications, safety and efficacy of lipofilling and concluded that, due to the limited data, they could not suggest evidence based recommendations concerning cancer recurrence (7). Few studies have focused on the oncological safety of lipofilling after BCS (8-10). Petit et al. reported the first case-matched retrospective series, which found no significant difference in local events (local relapse, locoregional relapse, or local relapse with synchronous metastases) between patients who underwent lipofilling after BCS and those who underwent BCS alone (10). Similarly, Gale et al. conducted a casematched retrospective series comparing oncological outcomes between 211 patients who underwent lipofilling after BCS with control patients matched for five variables and found no significant association between lipofilling and disease recurrence, both locoregional and distant (11). However, in 2013, Krastev et al. performed a systematic review and found marked increase in locoregional recurrence in patients who underwent lipofilling after BCS when compared to a large series of women treated with BCS (12). Despite the merits of these and other studies found in the literature, the lack of stratification of recurrence rate by tumor subtype, tumor stage, receptor status, and time to follow-up potentially limit their utility and applicability. The purpose of this project is to conduct a literature review utilizing PRISMA guidelines to investigate whether sufficient data exists to determine whether lipofilling following BCS increases the rate of locoregional tumor recurrence (LRR).

\section{Methods}

A systematic, online literature search was conducted following PRISMA guidelines (Figure 1). PubMed and MEDLINE were utilized to identify articles that investigated or commented on the oncological safety of lipofilling following BCS up until October 2019. Keywords included: "breast conserving surgery," "lumpectomy," "partial mastectomy," "lipofilling," "fat transfer," "fat grafting," "recurrence," and "safety". Oncoplastic surgery is a form of BCS $(13,14)$ and was included in our definition of BCS. Ninety-three articles were initially identified. The citations of the 7 review articles/meta-analyses acquired from our search were screened to identify 6 additional articles. Fiftyfour unique articles remained after removing duplicates. Articles met inclusion criteria if (I) the study participants underwent lipofilling following BCS and (II) the authors reported the recurrence rate. Exclusion criteria included (I) mastectomy or other types of surgery without any patients undergoing BCS prior to lipofilling; (II) non-cancer related reconstruction; (III) in vitro and animal studies; (IV) review articles and meta-analyses; (V) correspondence/commentary; (VI) full text unavailable; (VII) languages other than English without an available translation. Nineteen articles satisfied inclusion and exclusion criteria.

After excluding ineligible publications, nineteen articles underwent full-text analysis by two independent reviewers (SMC, YS) to extract information regarding the number of patients that specifically underwent BCS, average time to follow-up from lipofilling, the interval between primary resection and lipofilling, presence of a subgroup analysis for ER/PR/HER-2 status to determine if there was an association with recurrence, if the study design included a control, if the control was matched for ER/PR/Her-2, commentary on adequate powering, recurrence rates, statement of oncologic safety, and call for additional studies/trials. Lipofilling was considered "safe" if there was (I) a description of the interval between cancer resection and lipofilling; (II) a minimum follow-up period of 6 years after primary cancer resection; (III) a minimum follow-up period of 3 years after lipofilling; (IV) an analysis of breast 


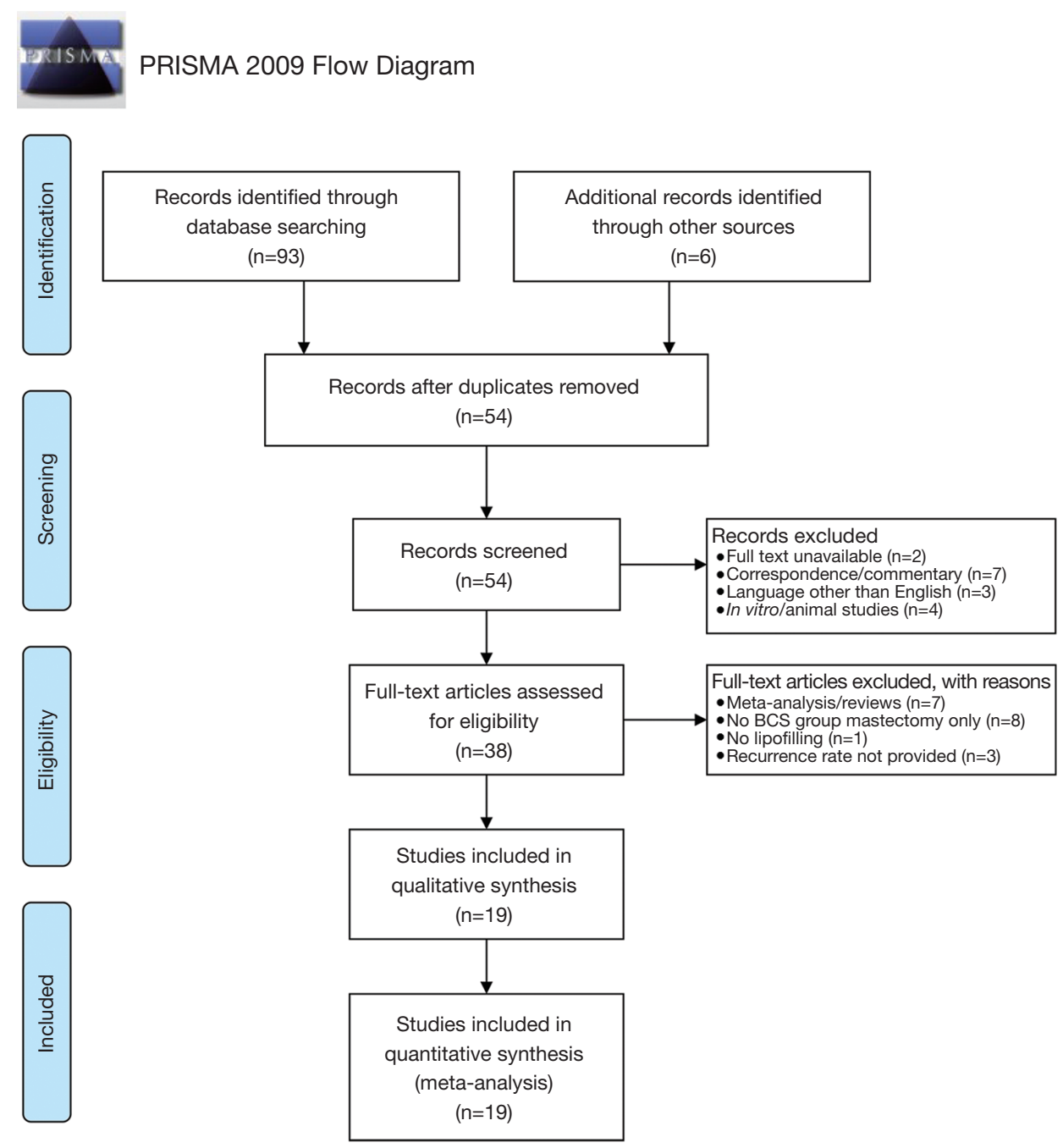

Figure 1 PRISMA methodology schematic.

cancer sub-groups specifically focusing on ER/PR/Her2; (V) a defined cohort comparison group; (VI) controls matched for receptor status; (VII) adequate powering.

The interval between the primary oncologic surgery and lipofilling needed to be defined, as immediate lipofilling poses the risk of having positive margins prior to transferring stem cells, and adequate time may be required to ensure there is no recurrence before introduction of these autologous cells. A follow-up time of 6 years from primary resection and 3 years from lipofilling was requisite to conclude whether lipofilling is safe since most locoregional recurrences occur between 3-6 years following BCS $(15,16)$. In addition, the ACOSOG Z0011 trial, which dramatically impacted the management of patients with axillary metastases, required a median time to follow-up of 6 years (17). It is critical to match for receptor status in a cohort study design. A sub-group analysis of receptor status and outcome after lipofilling is needed as specific types of cancers have different tendencies to recur (18).

\section{Results}

\section{Study selection}

Nineteen studies satisfied inclusion and exclusion criteria (Figure 1). Of the nineteen, none of the studies satisfied all 7 criteria required to conclude that fat grafting after BCS is safe (Figure 2). 


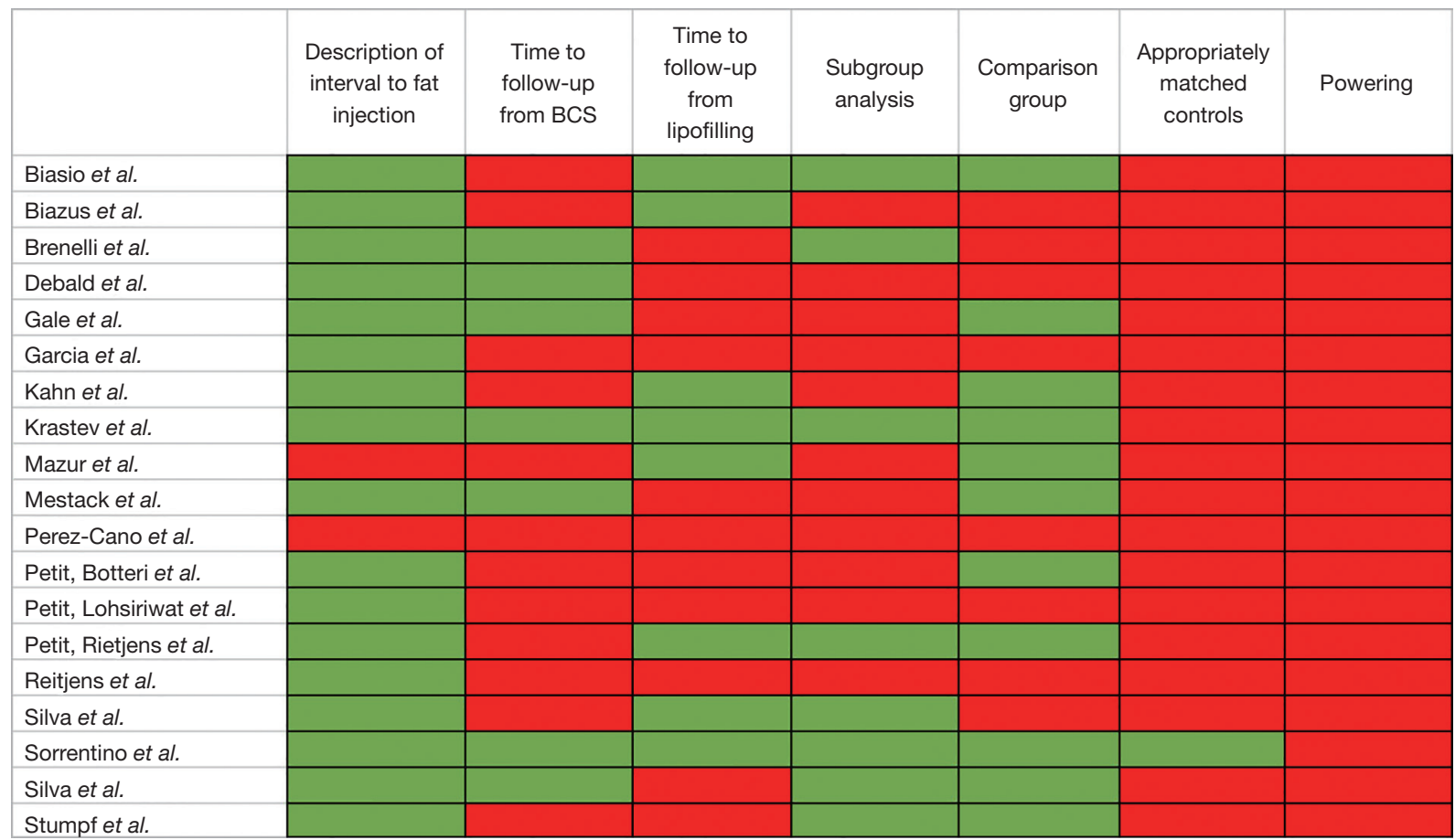

Figure 2 Graphic representation of required criteria to establish safety of lipofilling following BCS. Criteria to determine safety of lipofilling following BCS included: (I) description of the interval between BCS and lipofilling; (II) time to follow-up from BCS of 6 years; (III) time to follow-up from lipofilling of 3 years; (IV) subgroup analysis focusing on ER, PR, Her-2; (V) presence of any comparison group at all; (VI) comparison group matched controls specifically for ER, PR, and Her-2; (VII) adequate powering. BCS, breast conservation surgery.

\section{Participants}

The number of patients who underwent lipofilling following BCS ranged from 7-143 patients (Table 1). Krastev et al. found that 13 patients were diagnosed with a second primary tumor in the contralateral breast during oncologic followup and underwent additional lipofilling following tumor resection. Ipsilateral recurrence between primary resection and lipofilling was intended to be a contraindication to lipofill in groups not investigating the role of "immediate" lipofilling, however, but one patient in the study performed by Brenelli et al. experienced a locoregional recurrence and underwent subsequent lipofilling (19).

\section{Study characteristics}

Four retrospective case series, six prospective case series, six retrospective case control studies, and three prospective case control studies were included (Table 1).

\section{Comparison group}

Eleven studies included a comparison group (Table 2). Eight of the eleven studies were case control studies and three were retrospective case series in which patients who underwent BCS without lipofilling during the same time period at the same institution were selected as the comparison group without matching for any patient characteristics (Table 3). Three studies matched patients for ER receptor status alone $(10,11,20)$, two studies matched patients for both ER and PR (21,22), and one study by Sorrentino et al. matched patients for Luminal A subtype, Luminal B subtype, and Triple Negative disease (23).

Sorrentino et al. performed a sub-analysis examining fat grafting and locoregional recurrence and distant metastases showed no increase in rate of recurrence during follow-up. However, their predictive model demonstrates that after 80 months, there would be a statistically significant greater recurrence rate in patients with Luminal A cancers that 
Table 1 Study design and rates of locoregional recurrence (LRR)

\begin{tabular}{|c|c|c|c|c|c|}
\hline Study & Design & BCS + lipofilling (n) & BCS alone $(n)$ & LRR BCS + lipofilling (\%) & LRR BCS alone (\%) \\
\hline Biazus et al. & PCS & 65 & 0 & 7.86 & $\mathrm{n} / \mathrm{a}$ \\
\hline Brenelli et al. & PCS & 59 patients ( 75 cases) & 0 & $5.1^{*}$ & $\mathrm{n} / \mathrm{a}$ \\
\hline Debald et al. & RCS & 17 & 0 & 0 & $\mathrm{n} / \mathrm{a}$ \\
\hline Garcia et al. & PCS & 37 & 0 & 0 & $\mathrm{n} / \mathrm{a}$ \\
\hline Kahn et al. & PCS & 32 & 39 & 0 & 0 \\
\hline Krastev et al. & $\mathrm{RCC}$ & 139 & 150 & 0.72 & 4.7 \\
\hline Mazur et al. & PCC & 7 & 28 & $3.7^{\star *}$ & $4.1^{\star *}$ \\
\hline Petit, Botteri et al. & $\mathrm{RCC}$ & 125 & 250 & 1.0 & 4.0 \\
\hline Petit, Lohsiriwat et al. & RCS & 143 patients (170 cases) & 0 & 0.8 & $\mathrm{n} / \mathrm{a}$ \\
\hline Petit, Rietjens et al. & $\mathrm{RCC}$ & 59 & 118 & 0.18 & 0.3 \\
\hline Rietjens et al. & PCS & 77 & 0 & 0.01 & $\mathrm{n} / \mathrm{a}$ \\
\hline Silva et al. & RCS & 63 & 0 & 3.2 & $\mathrm{n} / \mathrm{a}$ \\
\hline Silva et al. & PCC & 58 & 124 & 1.7 & 2.4 \\
\hline Sorrentino et al. & PCC & 54 & 444 & $6.4^{\star \star}$ & $5.0^{\star \star}$ \\
\hline Stumpf et al. & RCC & 27 & 167 & 3.7 & 4.2 \\
\hline
\end{tabular}

${ }^{*}$, reported as \% recurrence per patient enrolled; ${ }^{* *}$, overall LRR, not stratified for only BCS (includes mastectomy). BCS, breast conservation surgery; RCS, retrospective case series; PCS, prospective case series; RCC, retrospective case control; PCC, prospective case control.

underwent lipofilling compared to the matched control Luminal A cancers that did not undergo lipofilling (23).

There was a statistically significant difference in Her-2 expression between study arms in two studies: Silva et al. 2017 included $19.5 \%$ Her-2 positive in the lipofilling group vs. $28 \%$ in the control group $(\mathrm{P}=0.022)(20)$; Gale et al. included $15 \%$ Her-2 positive in the lipofilling group vs. $7.3 \%$ in the control group $(\mathrm{P}=0.013)$, but $37 \%$ of patients in the lipofilling group and $52.4 \%$ of patients in the control group had an unknown Her-2 status (11). There was equal representation of triple negative disease, ER, PR, and HER-2 expression in the experimental arms inclusive of both BCS and mastectomy without stratification for BCS alone in the study performed by Krastev et al. (24).

\section{Time intervals}

Seventeen of the nineteen studies specified the interval between BCS and lipofilling. Five studies evaluated safety after immediate lipofilling, lipofilling performed during the same operative time as the primary resection (Table 2). There were two articles that mandated that patients were cancer free for 12 months after primary cancer surgery as a part of their inclusion criteria, but they did not specify the actual interval between surgery and fat injection $(25,26)$.

The overall average range in time from primary surgery to fat injection ranged from 0-76 months. The average time to follow-up after lipofilling to assess for recurrence ranged from 23 days- 60 months.

Eight studies out of nineteen had a three year time- 


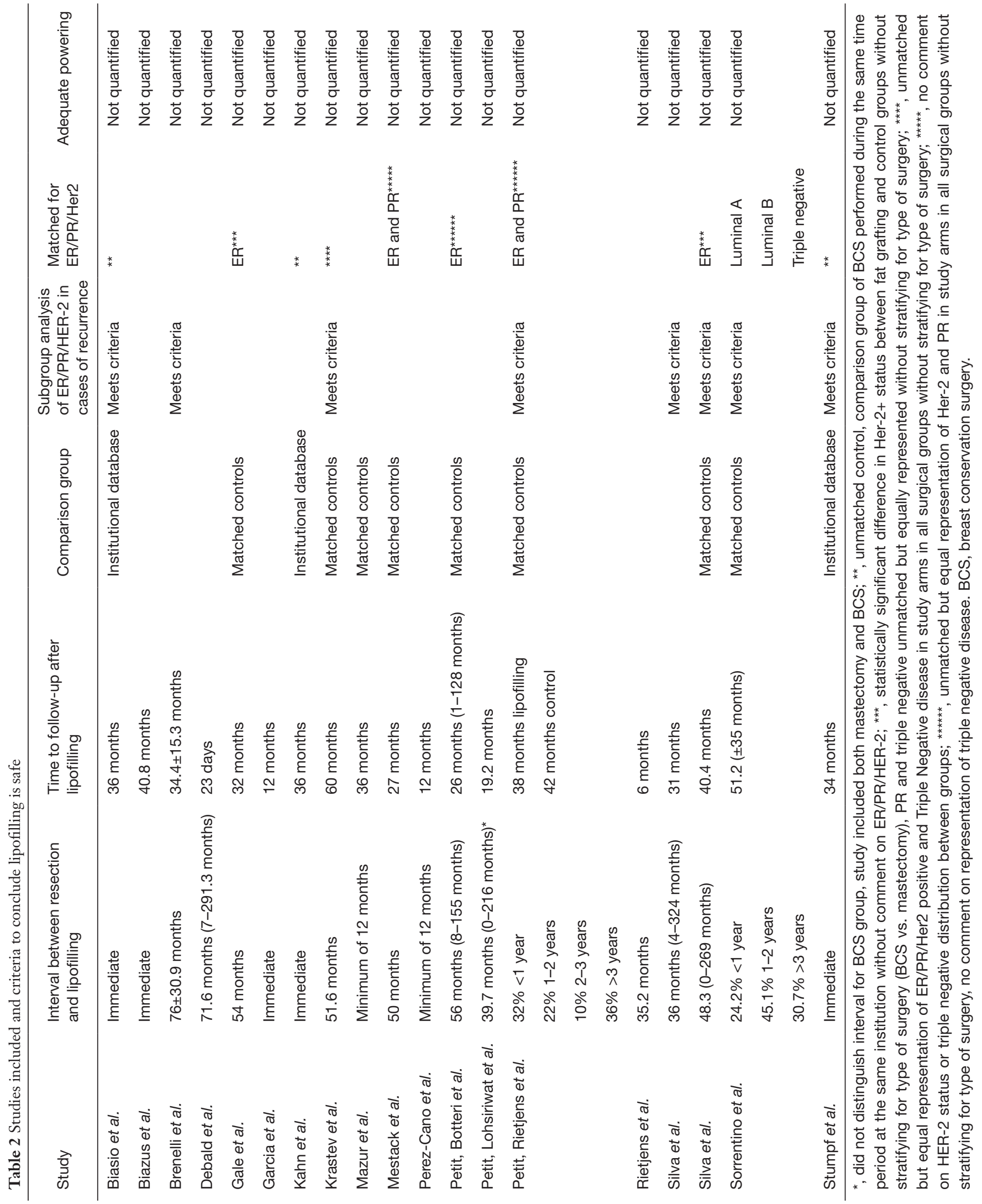


Table 3 Matched variables in studies including a comparison group

\begin{tabular}{|c|c|}
\hline Reference & Matching criteria \\
\hline Biasio et al. & Unmatched controls \\
\hline \multirow[t]{6}{*}{ Gale et al. } & Date of primary surgery (within 2 years) \\
\hline & Age (within 5 years) \\
\hline & Type of surgery (BCS vs. mastectomy) \\
\hline & Histology \\
\hline & ER status \\
\hline & Disease free interval before fat grafting \\
\hline Kahn et al. & Unmatched controls \\
\hline \multirow[t]{5}{*}{ Krastev et al. } & Age \\
\hline & Type of surgery (BCS vs. mastectomy) \\
\hline & Tumor invasiveness \\
\hline & Disease stage \\
\hline & Disease free interval before fat grafting \\
\hline \multirow[t]{3}{*}{ Mazur et al. } & Year of admission to a single institution \\
\hline & Age \\
\hline & Histology \\
\hline \multirow[t]{8}{*}{ Mestack et al. } & Date of primary surgery \\
\hline & Date of fat grafting \\
\hline & Histology \\
\hline & ER status \\
\hline & PR status \\
\hline & Adjuvant hormone therapy \\
\hline & Disease free interval after primary surgery \\
\hline & Disease free interval after fat grafting \\
\hline \multirow[t]{6}{*}{ Petit, Botteri et al. } & Age (within 5 years) \\
\hline & Year of surgery (within 2 years) \\
\hline & Type of surgery (BCS vs. mastectomy) \\
\hline & Histology \\
\hline & Tumor size (T1/2/3) \\
\hline & ER status \\
\hline
\end{tabular}

Table 3 (continued)

to-follow-up after lipofilling (22-25,27-30), seven studies satisfied an average time to follow-up after BCS of at least six years (11,19-21,23,24,31). Krastev et al. and Sorrentino et al. satisfied both the time to follow-up from BCS and from
Table 3 (continued)

\begin{tabular}{|c|c|}
\hline Reference & Matching criteria \\
\hline \multirow[t]{8}{*}{ Petit, Rietjens et al. } & Age (within 5 years) \\
\hline & Year of surgery (within 2 years) \\
\hline & $\begin{array}{l}\text { Type of surgery (BCS vs. mastectomy) } \\
\text { histology }\end{array}$ \\
\hline & ER status \\
\hline & PR status margin status \\
\hline & Hormonal therapy \\
\hline & Radiotherapy \\
\hline & Disease free interval after primary surgery \\
\hline \multirow[t]{6}{*}{ Silva et al. 2017} & Age (within 5 years) \\
\hline & Year of surgery (within 3 years) \\
\hline & Type of surgery (BCS vs. mastectomy), \\
\hline & Histology \\
\hline & Lymphatic involvement \\
\hline & ER status \\
\hline \multirow[t]{6}{*}{ Sorrentino et al. } & Age \\
\hline & Histopathology \\
\hline & Stage \\
\hline & Luminal A (ER+ PR+ HER2-) \\
\hline & Luminal B (ER+ PR+ HER2-) \\
\hline & Triple negative \\
\hline Stumpf et al. & Unmatched controls \\
\hline
\end{tabular}

lipofilling, Krastev et al. followed patients for an average of 9 years from BCS and 4.3 years from lipofilling $(23,24)$. Sorrentino et al. followed patients for an average of 6.2 years from BCS and 4.2 years from lipofilling (Figure 2). One of the subgroups within the Petit, Reitjens et al. study may also satisfy both criteria, as $36 \%$ of their study participants underwent lipofilling $>36$ months after primary cancer resection and the average time to follow-up after lipofilling was 38 months (22).

None of the "immediate" lipofilling studies satisfied our required time to follow-up after primary cancer resection since there was no waiting period between resection and lipofilling. However, all of the immediate lipofilling studies with the exception of Garcia et al., satisfied a requisite 3-year follow-up time following fat grafting (32). Of the other 
immediate lipofilling studies, Biasio, Biazus et al., Kahn et al., and Stumpf et al. had a time to follow-up from the BCS and immediate lipofilling of 36, 40, 36 and 34 months respectively (27-29,33).

\section{Sub-group analysis for receptor status}

Eight studies reported the ER/PR/Her-2 status of cases of locoregional recurrence as a part of their subgroup analysis (Figure 2). Only one looked at Her-2 enriched and triple negative disease (23). Sorrentino et al. did not find a statistically significant difference in LRR upon subgroup analysis for Luminal A, Luminal B, Her-2 enriched, or Triple Negative disease in lipofilling compared to control groups. However, their logistic regression model found a statistically significant difference in locoregional recurrence after 80 months for Luminal-A subtype. This analysis was not restricted to BCS and included patients undergoing mastectomy.

\section{Recurrence}

Petit, Reitjens et al. demonstrated a significant difference in the locoregional recurrence rates between patients undergoing lipofilling after BCS compared to BCS alone, $0.18 \%$ and $0.03 \%$ respectively (Table 1 ). Five studies reported a locoregional recurrence rate of $0 \%$ in the fat grafting group. Krastev et al. and Sorrentino et al. found that there was a higher rate of locoregional recurrence in the control group than the fat grafting group after BCS (Table 1), but that the difference was not significant. Krastev et al. found a statically significant difference in overall mortality in the control group compared lipofilling group in all forms of surgery (both mastectomy and BCS) of $11.0 \%$ in the control group compared to $2.6 \%$ in the lipofilling group $(\mathrm{P}<0.001)$. This difference persisted when the analysis was restricted to breast cancer-specific mortality (24).

\section{Statement of oncological safety}

Fifteen papers determined that further investigation is required to determine if there is an association between lipofilling after BCS and recurrence. Five articles stated that it is unclear whether or not lipofilling is safe. There were seven studies that simultaneously declared that there was a need for further investigation but endorsed that lipofilling is "safe" $(20,21,25,26,30,31,33)$. Four utilized ambiguous verbiage such as "seems to be safe," or "interpret with other similar case-controlled studies" $(10,11,27,28)$. Petit, Rietjens and Botteri concluded that there is an increased risk in certain groups and that lipofilling may be unsafe for those patients (22).

\section{Statistical power}

None of the studies quantified the statistical power of their analyses.

\section{Risk factors for recurrence}

Biazus, Stumpf, Melo et al. performed a subgroup analysis that demonstrated that the number of metastatic axillary lymph nodes was associated with increased recurrence after lipofilling (28). Petit, Botteri, Lohsiriwat et al. found a higher risk of recurrence in patients with intraepithelial neoplasia and not invasive disease (10). Petit, Rietjens, and Botteri found that Ki-67 expression, age $<50$, and highgrade neoplasia were associated with increased rates of recurrence (22). Silva-Vergara, Fontdevila et al. found that there was an increased risk of recurrence if lipofilling was performed within 3 months of primary resection (20). Krastev et al. found no significant difference in local recurrence rates based on receptor subtype or if the injection interval was $<5$ years from primary resection to lipofilling (24).

Sorrentino et al. did not find that there was a statistically significant increase in LRR based on tumor subtype during their time to follow-up but their logistic regression model found a statistically significant difference in locoregional recurrence after 80 months for patients with Luminal A type cancer.

\section{Discussion}

The importance of this study cannot be understated. Lipofilling in the setting of breast conservation is presently being done and the data presented here shows, at best, that the oncologic safety supporting this technique in this population of patients is unclear. Surgeons and their patients performing this type of reconstruction need to know the potential unclear implications of injecting stem cells into a breast defect that has had a history of cancer present. Several studies have attempted to address the safety of lipofilling, with some confusingly concluding that lipofilling is safe yet still proceeding to recommend for future studies to investigate for safety $(20,21,23,25,26,29,30)$. Our criteria 
for safety was adopted from past studies that addressed oncologic safety based on interventions performed. With regards to our first criteria, seventeen of the nineteen studies specified the average time interval between resection and lipofilling. Average values reflect a range of time intervals, raising the possibility that some study participants had adequate time ( $>6$ years) to follow-up from primary surgery while others did not. The wide ranges in injection intervals between studies demonstrates a lack of consensus for a "safe" time of injection. It can be argued that the time to injection may affect the response of local cells to the stem cell effect of fat being injected. Silva-Vergara et al. demonstrated that there was an increased risk of recurrence if lipofilling occurred within three months after partial mastectomy (30). On the other hand, Krastev et al. found that there was not an increased risk of recurrence when injection intervals were $<5$ years (24) and Sorrentino et al. found early fat grafting was not associated statistically significant increased rates of recurrence despite finding that there was a higher proportion of early fat grafting among recurrent patients (23). Brenelli et al. reported a locoregional recurrence risk of $5.1 \%$ and only included 3 patients of the 59 that underwent lipofilling following BCS in their locoregional recurrence calculation rather than the 4 that experienced a recurrence since one patient actually had a histologically confirmed tumor recurrence in the interval prior to lipofilling (24).

Immediately injecting fat into a partial mastectomy cavity without proven negative margins could be dangerous if a positive margin would receive stem cells from injected fat. There have been retrospective studies that have injected fat into partial mastectomy defects at the same time of oncologic resection which is not recommended by the authors without clear future prospective randomized data and patient-informed consent proving safety $(28,29,32,33)$. Krastev et al. and Sorrentino et al. were the only groups that employed an experimental design with an adequate time to follow-up from BCS and from lipofilling, but their methodology and results were still imperfect and plagued with design flaws that shall be discussed $(23,24)$.

Locoregional recurrence was studied in all included articles, but a majority of studies underpowered their comparison groups, especially since the number of subjects became too few if breast cancers were separated into their molecular subtypes (based on the ER, PR and Her2 status). Of the 11 studies that had a comparison group, only 3 studies matched patients based on both ER and PR status $(21,22)$. Krastev et al. did attempt to investigate the role of hormone receptor status and triple negative disease and found no significant difference in locoregional recurrence between lipofilling and control groups (24). However, this study mixed mastectomy patients with breast conservation patients when looking at molecular subtypes between the lipofilling and control groups. Sorrentino et al. matched study participants by breast cancer subtype: Luminal A, Luminal B, Her-2 enriched, and Triple Negative disease. However, they did not match patients for the type of surgery performed. In fact, mastectomy accounted for $76.8 \%$ of lipofilling arm whereas only $25.6 \%$ of the controls underwent mastectomy and the remainder underwent BCS. Given this mixture of mastectomy and BCS patient sampling, even though they did perform a sub-group analysis, one cannot say with statistical certainty that receptor status does not impact recurrence after BCS when cancerous cells may still persist in the tumor cavity.

Without adequate powering for the different molecular subtype presentations between the lipofilling and control groups specifically undergoing BCS, a Type II statistical error is possible showing no statistical difference between the lipofilling and no lipofilling arms when in fact there may be, as chance plays a large role in the conclusion. To date, there is no study with confirmed, adequate powering looking at lipofilling after breast conservation when separating breast cancers into their molecular subtypes.

Although Krastev et al. did not find a difference in locoregional recurrence in the cases of lipofilling after BCS, the overall mortality for patients undergoing both BCS and mastectomy without lipofilling was $11.0 \%$ while the overall mortality in lipofilling group was $2.6 \%$. This recurrence rate is high considering they did not enroll any patients with stage IV disease, and the majority of their patients had either stage 0 or early stage disease. The 5 -year breast cancer specific survival for stage 1 disease has been noted to be between $98-100 \%$ according to the literature (34). Thus, it is highly unlikely that fat grafting lead to a more favorable prognosis and further investigation into pre-selection biases or confounding variables is critical. In contradiction, Petit et al. found that lipofilling was associated with an increase of local recurrence when intraepithelial neoplasia (e.g., DCIS) was present as opposed to invasive disease (10).

There have been transitional studies utilizing animal models to explore the safety of lipofilling which also have had mixed conclusions $(35,36)$. Tsuji et al. and Silva et al. both utilized "immortal" cell lines injected into rats to simulate a tumor bed prior to fat transfer. They found that autologous fat transfer did not encourage tumor 
growth, and may actually suppress tumor cell proliferation. However, clonal cell lines do not reproduce the tumor heterogeneity that is present in vivo, and surgery was not performed in either study. In fact, Silva et al. suggested that mechanical pressure of the adipose cells injected adjacent to the tumors may have impaired tumor cell proliferation.

In vitro models have associated fat grafting with increased tumor growth. Almarzougi et al. examined the role of lipoaspirates and resected abdominal fat on tumor cells and found that adipose increases the proliferation of MCF-7 (ER/PR positive, luminal type) cells, contrary to the findings in the Tsuji et al. animal model (37). Moreover, Massa et al. demonstrated that adipose fat grafts could accelerate development a subclinical tumor or support locoregional recurrence of a previous tumor (38).

Historically, past surgical interventions were studied using strict experimental design requirements $(17,39,40)$ that are absent from the studies so far that have investigated lipofilling. The authors submit that the same rigor of experimental design that would include appropriate molecular subtype analysis with adequate powering, an established follow-up period, and an ethical, informed consent process given prospectively to the patient should be required before declaring lipofilling as safe in breast conservation. Fat grafting may be useful in certain clinical scenarios, but if it was found to be harmful in partial mastectomy defect corrections, it is possible that there could be subsequent reactionary regulations limiting the ability to fat graft in all facets of reconstruction.

The data supporting the "safety" of lipofilling in breast conservation so far are incomplete; thus, one cannot state that lipofilling in breast conservation is safe. At best, these results show that the safety of lipofilling in breast conservation is unclear with contradictory data present in the literature. Surgeons with interest in fat grafting and lipofilling need to be cautious and inform their patients requesting these interventions of these uncertainties regarding loco-regional recurrences. Central to our Hippocratic Oath is to do no harm and with this, regardless of how nicely lipofilling corrects poor aesthetics after partial mastectomy, form can never trump oncologic safety. In a perfect world, there is a need for prospective studies with adequately powered cohort control groups looking at molecular subtypes and initiating lipofilling treatments at appropriate time intervals after margins are clear with appropriate post-operative follow-up in order to prioritize patient safety.

\section{Conclusions}

Present studies looking at lipofilling in the setting of breast conservation have several design limitations and do not prove oncologic safety. A well designed, ethical, appropriately powered, prospective study with comparison groups looking at molecular subtypes needs to be performed to ensure patient safety before surgeons consider treating breast conservation defects with lipofilling.

\section{Acknowledgments}

None.

\section{Footnote}

Conflicts of Interest: The authors have no conflicts of interest to declare.

Ethical Statement: The authors are accountable for all aspects of the work in ensuring that questions related to the accuracy or integrity of any part of the work are appropriately investigated and resolved.

\section{References}

1. Jonczyk MM, Jean J, Graham R, et al. Surgical trends in breast cancer: a rise in novel operative treatment options over a 12 year analysis. Breast Cancer Res Treat 2019;173:267-74.

2. Chatterjee A, Gass J, Burke MB, et al. Results from the American Society of Breast Surgeons Oncoplastic Surgery Committee 2017 Survey: Current Practice and Future Directions. Ann Surg Oncol 2018;25:2790-4.

3. Bircoll M. Cosmetic breast augmentation utilizing autologous fat and liposuction techniques. Plast Reconstr Surg 1987;79:267-71.

4. Vona-Davis L, Rose DP. Adipokines as endocrine, paracrine, and autocrine factors in breast cancer risk and progression. Endocr Relat Cancer 2007;14:189-206.

5. Lamszus K, Jin L, Fuchs A, et al. Scatter factor stimulates tumor growth and tumor angiogenesis in human breast cancers in the mammary fat pads of nude mice. Lab Invest 1997;76:339-53.

6. Lohsiriwat V, Curigliano G, Rietjens M, et al. Autologous fat transplantation in patients with breast cancer: 
"silencing" or "fueling" cancer recurrence? Breast 2011;20:351-7.

7. Gutowski KA; ASPS Fat Graft Task Force. Current applications and safety of autologous fat grafts: a report of the ASPS fat graft task force. Plast Reconstr Surg 2009;124:272-80.

8. Petit JY, Lohsiriwat V, Clough KB, et al. The oncologic outcome and immediate surgical complications of lipofilling in breast cancer patients: a multicenter study-Milan-Paris-Lyon experience of 646 lipofilling procedures. Plast Reconstr Surg 2011;128:341-6.

9. Cordeiro PG. Discussion: The oncologic outcome and immediate surgical complications of lipofilling in breast cancer patients: a multicenter study--Milan-Paris-Lyon experience of 646 lipofilling procedures. Plast Reconstr Surg 2011;128:347-8.

10. Petit JY, Botteri E, Lohsiriwat V, et al. Locoregional recurrence risk after lipofilling in breast cancer patients. Ann Oncol 2012;23:582-8.

11. Gale KL, Rakha EA, Ball G, et al. A case-controlled study of the oncologic safety of fat grafting. Plast Reconstr Surg 2015;135:1263-75.

12. Krastev TK, Jonasse Y, Kon M. Oncological safety of autologous lipoaspirate grafting in breast cancer patients: a systematic review. Ann Surg Oncol 2013;20:111-9.

13. Losken A, Hart AM, Chatterjee A. Updated Evidence on the Oncoplastic Approach to Breast Conservation Therapy. Plast Reconstr Surg 2017;140:14S-22S.

14. Chatterjee A, Gass J, Patel K, et al. A Consensus Definition and Classification System of Oncoplastic Surgery Developed by the American Society of Breast Surgeons. Ann Surg Oncol 2019;26:3436-44.

15. Botteri E, Bagnardi V, Rotmensz N, et al. Analysis of local and regional recurrences in breast cancer after conservative surgery. Ann Oncol 2010;21:723-8.

16. Bernardi S, Bertozzi S, Londero AP, et al. Incidence and risk factors of the intraoperative localization failure of nonpalpable breast lesions by radio-guided occult lesion localization: a retrospective analysis of 579 cases. World J Surg 2012;36:1915-21.

17. Giuliano AE, Hunt KK, Ballman KV, et al. Axillary dissection vs no axillary dissection in women with invasive breast cancer and sentinel node metastasis: a randomized clinical trial. JAMA 2011;305:569-75.

18. Pata G, Guaineri A, Bianchi A, et al. Long-Term Outcomes of Immunohistochemically Defined Subtypes of Breast Cancer Less Than or Equal to $2 \mathrm{~cm}$ After BreastConserving Surgery. J Surg Res 2019;236:288-99.
19. Brenelli F, Rietjens M, De Lorenzi F, et al. Oncological safety of autologous fat grafting after breast conservative treatment: a prospective evaluation. Breast J 2014;20:159-65.

20. Silva-Vergara C, Fontdevila J, Weshahy O, et al. Breast Cancer Recurrence Is not Increased With Lipofilling Reconstruction: A Case-Controlled Study. Ann Plast Surg 2017;79:243-8.

21. Mestak O, Hromadkova V, Fajfrova M, et al. Evaluation of Oncological Safety of Fat Grafting After BreastConserving Therapy: A Prospective Study. Ann Surg Oncol 2016;23:776-81.

22. Petit JY, Rietjens M, Botteri E, et al. Evaluation of fat grafting safety in patients with intraepithelial neoplasia: a matched-cohort study. Ann Oncol 2013;24:1479-84.

23. Sorrentino L, Regolo L, Scoccia E, et al. Autologous fat transfer after breast cancer surgery: An exact-matching study on the long-term oncological safety. Eur J Surg Oncol 2019;45:1827-34.

24. Krastev T, van Turnhout A, Vriens E, et al. Long-term Follow-up of Autologous Fat Transfer vs Conventional Breast Reconstruction and Association With Cancer Relapse in Patients With Breast Cancer. JAMA Surg 2019;154:56-63.

25. Mazur S, Zolocinska A, Siennicka K, et al. Safety of adipose-derived cell (stromal vascular fraction - SVF) augmentation for surgical breast reconstruction in cancer patients. Adv Clin Exp Med 2018;27:1085-90.

26. Perez-Cano R, Vranckx JJ, Lasso JM, et al. Prospective trial of adipose-derived regenerative cell (ADRC)enriched fat grafting for partial mastectomy defects: the RESTORE-2 trial. Eur J Surg Oncol 2012;38:382-9.

27. Biasio F, Bertozzi S, Londero AP, et al. Surgical and oncological outcomes of free dermal fat graft for breast reconstruction after breast-conserving surgery. Adv Clin Exp Med 2018;27:773-80.

28. Biazus JV, Stumpf CC, Melo MP, et al. Breast-Conserving Surgery with Immediate Autologous Fat Grafting Reconstruction: Oncologic Outcomes. Aesthetic Plast Surg 2018;42:1195-201.

29. Khan LR, Raine CR, Dixon JM. Immediate lipofilling in breast conserving surgery. Eur J Surg Oncol 2017;43:1402-8.

30. Silva-Vergara C, Fontdevila J, Descarrega J, et al. Oncological outcomes of lipofilling breast reconstruction: 195 consecutive cases and literature review. J Plast Reconstr Aesthet Surg 2016;69:475-81.

31. Debald M, Pech T, Kaiser C, et al. Lipofilling effects 
after breast cancer surgery in post-radiation patients: an analysis of results and algorithm proposal. Eur J Plast Surg 2017;40:447-54.

32. Moltó García R, González Alonso V, Villaverde Doménech $\mathrm{ME}$. Fat grafting in immediate breast reconstruction. Avoiding breast sequelae. Breast Cancer 2016;23:134-40.

33. Stumpf CC, Biazus JV, Zucatto FSÂE, et al. Immediate reconstruction with autologous fat grafting: influence in breast cancerregional recurrence. Rev Col Bras Cir 2017;44:179-86.

34. Weiss A, Chavez-MacGregor M, Lichtensztajn DY, et al. Validation Study of the American Joint Committee on Cancer Eighth Edition Prognostic Stage Compared With the Anatomic Stage in Breast Cancer. JAMA Oncol 2018;4:203-9.

35. Tsuji W, Valentin JE, Marra KG, et al. An Animal Model of Local Breast Cancer Recurrence in the Setting of Autologous Fat Grafting for Breast Reconstruction. Stem

Cite this article as: Cohen S, Sekigami Y, Schwartz T, Losken A, Margenthaler J, Chatterjee A. Lipofilling after breast conserving surgery: a comprehensive literature review investigating its oncologic safety. Gland Surg 2019;8(5):569-580. doi: $10.21037 /$ gs.2019.09.09
Cells Transl Med 2018;7:125-34.

36. Silva MMA, Kokai LE, Donnenberg VS, et al. Oncologic Safety of Fat Grafting for Autologous Breast Reconstruction in an Animal Model of Residual Breast Cancer. Plast Reconstr Surg 2019;143:103-12.

37. Almarzouqi F, Rennekampff HO, Stromps JP, et al. The effect of lipoaspirates vs. dissected abdominal fat on breast cancer cells in vitro. Eur J Med Res 2017;22:10.

38. Massa M, Gasparini S, Baldelli I, et al. Interaction Between Breast Cancer Cells and Adipose Tissue Cells Derived from Fat Grafting. Aesthet Surg J 2016;36:358-63.

39. Chagpar AB, Killelea BK, Tsangaris TN, et al. A Randomized, Controlled Trial of Cavity Shave Margins in Breast Cancer. N Engl J Med 2015;373:503-10.

40. Boughey JC, Suman VJ, Mittendorf EA, et al. Sentinel lymph node surgery after neoadjuvant chemotherapy in patients with node-positive breast cancer: the ACOSOG Z1071 (Alliance) clinical trial. JAMA 2013;310:1455-61. 